\title{
Aspects spécifiques de la radioprotection autour d'un cyclotron
}

\author{
N. BRASSART*, J. TUYN**, J. HERAULT*, P. CHAUVEL*
}

(Manuscrit reçu le 20 septembre 1993,

révisé le 16 novembre 1994, accepté le 19 janvier 1995)

RÉSUMÉ Ce rapport fait la synthèse des calculs de radioprotection effectués pour l'ensemble du bâtiment abritant le cyclotron biomédical de Nice et les salles de traitement en proton et neutron. Il n'a pas, bien entendu, la prétention d'évoquer tous les aspects de la sécurité et de la radioprotection autour des cyclotrons. Nous justifierons les épaisseurs des protections autour de la machine et des salles de traitement en indiquant les formules utilisées parmi les différentes méthodes décrites dans la littérature. Nous donnerons les caractéristiques des labyrinthes d'accès aux salles de traitement qui remplacent les lourdes portes trop traumatisantes pour les malades et délicates d'utilisation en fonctionnement médical. Nous discuterons de l'activation de l'air due essentiellement aux réactions des neutrons avec les différents éléments qui le constituent et de la nécessité d'une ventilation dans les salles de traitement, la voie de faisceau et le hall du cyclotron.

ABSTRACT The radiation protection calculations used to determine the wall thicknesses of the structure housing the accelerator, the proton and the neutron treatment areas at the Biomedical cyclotron of Nice are presented. The results were obtained from different equations and methods taken from the literature. The paper gives the characteristics of the entrance labyrinths to the treatment rooms. These labyrinths allowed the suppression of heavy motorized doors which tend to slow access and frighten patients. The problem of radioactivity of air caused mainly by interactions of neutrons with the different air compounds are discussed. The necessary ventilation system for the cyclotron bunker, the beam line and the treatment rooms is described. This paper does not address all the aspects of radiation protection and safety precautions for cyclotrons.

\section{Introduction}

On abordera les aspects essentiels de la radioprotection autour d'un cyclotron en considérant plus spécifiquement le cas d'un cyclotron biomédical tel que Medicyc, installé à Nice : il accélère jusqu'à une énergie de $65 \mathrm{MeV}$ des ions $\mathrm{H}^{-}$qui sont extraits du cyclotron par épluchage de leurs électrons à la traversée d'une mince feuille de carbone. Le flux de protons ainsi produit est guidé par les différents aimants de la voie de faisceau jusqu'aux deux salles de traitement, protonthérapie et neutronthérapie.

* Centre Antoine Lacassagne, Cyclotron biomédical, 227 avenue de la Lanterne, 06200 Nice, France.

** CERN, CH-1211 Genève, Suisse. 


\section{Les causes de la radioactivité}

Lorsqu'un proton interagit avec la matière, il perd de l'énergie par ionisation et collision nucléaire. Cette ionisation est maximale à la fin de son trajet appelé parcours ; ce dernier dépend de l'énergie des protons et du matériau traversé. A une énergie de $65 \mathrm{MeV}$, ce parcours est de $3,4 \mathrm{~cm}$ dans l'eau, $1,9 \mathrm{~cm}$ dans le béton, et $6,3 \mathrm{~mm}$ dans le cuivre. Ce faisceau primaire est donc très vite arrêté et, du point de vue de la radioprotection, seuls sont donc à prendre en compte, à cette énergie, les flux de neutrons et de photons émis lors de l'interaction de ce faisceau de protons sur les différentes cibles qu'il peut rencontrer.

\subsection{Machine en fonctionnement}

\subsubsection{Les différentes cibles}

Lors de l'accélération des ions, de leur extraction ou de leur transport, le faisceau de protons rencontre les différentes structures du cyclotron et de la voie de faisceau telles que les dés d'accélération en cuivre, les aimants, les quadrupôles en fer et les tubes à vide en acier ainsi que différentes structures en aluminium, cuivre et fer dans les salles de traitement. Pour mesurer leur intensité, les protons sont arrêtés en plusieurs endroits de la voie de faisceau sur des cages de Faraday faites de plaques de tantale de $6 \mathrm{~mm}$ d'épaisseur. Pour la protonthérapie, le faisceau est diffusé sur une feuille de tantale de $0,05 \mathrm{~mm}$ d'épaisseur. Le flux de neutrons nécessaire aux traitements est obtenu en bombardant une cible de béryllium de $17 \mathrm{~mm}$ d'épaisseur.

\subsubsection{L'activation de l'air}

Dans les salles où les réactions précédentes se produisent, l'interaction des neutrons avec les différents constituants de l'air entraîne la production dans l'air d'isotopes radioactifs. Il est important que les débits de dose dus à cette activation de l'air restent inférieurs aux limites recommandées en radioprotection, non seulement pour le personnel, mais aussi pour la population vivant autour du site.

\subsection{Machine à l'arrêt}

L'activité induite, qui résulte de la décroissance de la radioactivité dans les différentes structures rencontrées par le faisceau, est à prendre en compte lors des interventions de maintenance de la machine et du transport de certaines pièces. Les caractéristiques de cette radioactivité dépendent du type et de l'énergie des particules accélérées, de l'intensité du faisceau et des matériaux irradiés (Tab. I). 
TABLEAU I

Radionucléides couramment identifiés dans les matériaux solides irradiés autour des accélérateurs Radionuclides commonly identified in solid materials irradiated around accelerators

\begin{tabular}{|c|c|}
\hline \multicolumn{2}{|c|}{ 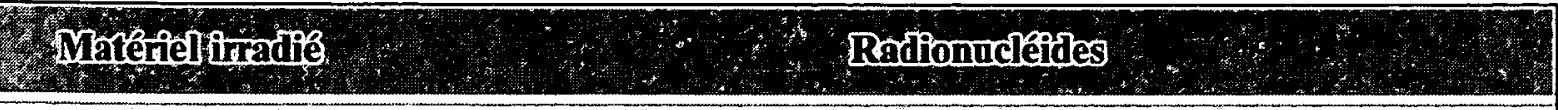 } \\
\hline Plastiques, huiles & ${ }^{7} \mathrm{Be},{ }^{11} \mathrm{C}$ \\
\hline Béton, aluminium & ci-dessus plus ${ }^{22} \mathrm{Na},{ }^{24} \mathrm{Na},{ }^{32} \mathrm{P},{ }^{42} \mathrm{~K},{ }^{45} \mathrm{Ca}$ \\
\hline Fer, acier & $\begin{array}{l}\text { ci-dessus plus }{ }^{44} \mathrm{Scm},{ }^{46} \mathrm{Sc},{ }^{47} \mathrm{Sc},{ }^{48} \mathrm{Sc},{ }^{48} \mathrm{~V},{ }^{51} \mathrm{Cr},{ }^{52} \mathrm{Mn},{ }^{52} \mathrm{Mn} \\
{ }^{54} \mathrm{Mn},{ }^{56} \mathrm{Mn},{ }^{57} \mathrm{Co},{ }^{58} \mathrm{Co},{ }^{60} \mathrm{Co},{ }^{57} \mathrm{Ni},{ }^{55} \mathrm{Fe},{ }^{59} \mathrm{Fe}\end{array}$ \\
\hline Cuivre & ci-dessus plus ${ }^{65} \mathrm{Ni},{ }^{61} \mathrm{Cu},{ }^{64} \mathrm{Cu},{ }^{63} \mathrm{Zn},{ }^{65} \mathrm{Zn}$ \\
\hline
\end{tabular}

D'après [7].

\section{Calcul des protections}

\subsection{Préambule}

Le faisceau primaire monoénergétique produit, après interaction avec les différentes structures, un champ complexe d'une grande quantité de particules et d'une large gamme d'énergie. Ce spectre est d'autant plus intense que l'énergie et surtout l'intensité du faisceau initial de particules sont élevés. Il semble, à la suite d'expériences faites autour de cyclotrons, notamment à Berkeley [1, p. 65], que l'on puisse établir comme règle générale le fait que $50 \%$ de la dose équivalente dans le champ d'irradiation est due aux neutrons d'énergie comprise entre 0,1 et $10 \mathrm{MeV}, 10$ à $20 \%$ aux neutrons lents et au rayonnement gamma, le complément à des neutrons d'énergie supérieure à $10 \mathrm{MeV}$.

Les épaisseurs des protections ne sont donc pas déterminées par le parcours des particules initiales mais par celui des particules secondaires, essentiellement des neutrons et, dans une moindre mesure, des photons. Elles doivent être suffisantes pour que le personnel puisse travailler en toute sécurité autour des installations du cyclotron de la voie de faisceau et des salles de traitement en respectant les normes de radioprotection en vigueur en France [1].

Les matériaux utilisés pour la construction des bâtiments sont aussi le siège des réactions évoquées précédemment avec les protons et les neutrons et devront donc être choisis de telle sorte que leur activation soit la plus faible possible : par exemple, le sable et les agrégats entrant dans la fabrication du béton contiennent des proportions de ${ }^{23} \mathrm{Na}$ et ${ }^{55} \mathrm{Mn}$ différentes suivant leur provenance ; or, la capture de neutrons lents par ces composants produit des 
isotopes radioactifs ${ }^{24} \mathrm{Na}$ et $56 \mathrm{Mn}$ qui, s'ils sont en quantité trop importante, entraînent une irradiation du personnel que l'on peut facilement éviter en choisissant un béton à faible teneur en ${ }^{23} \mathrm{Na}$ et ${ }^{55} \mathrm{Mn}$. Une analyse par activation a été faite au CERN sur des échantillons de composants du béton provenant de différents sites afin de choisir ceux utilisés pour la construction des bâtiments du cyclotron à Nice.

\subsection{Formulation générale}

Nous considérons, essentiellement, la production de neutrons par action des protons sur cible épaisse de cuivre. D'après différents auteurs [2, 7, p. 461] les résultats ne sont pas très différents avec les cibles de fer ou d'aluminium. Par contre, le flux de neutrons obtenu sur cible de béryllium est 2,5 fois supérieur à celui obtenu à partir d'une cible de cuivre.

Quand des protons de haute énergie sont arrêtés sur une cible épaisse, les neutrons émis appartiennent à deux spectres distincts : neutrons d'évaporation de faible énergie inférieure à $10 \mathrm{MeV}$ et de distribution angulaire isotrope et neutrons de cascade qui ont un spectre continu d'énergie et dont l'énergie maximale est celle du proton incident. Le développement de cascades intranucléaires commence à devenir important au-delà de $50 \mathrm{MeV}[1, \mathrm{p} .213]$.

Le calcul de l'épaisseur des protections a été fait en utilisant la formule simplifiée suivante décrite par Braid [3], Tesch [12] et Stevenson [9].

$$
H=\frac{H_{0} \exp (-x / \lambda)}{r^{2}}
$$

$H_{0}=$ équivalent de dose à $1 \mathrm{~m}$ de la cible en l'absence de protection (Sv.m²/proton)

$H$ = équivalent de dose à une distance $r$ de la cible derrière les protections (Sv/proton)

$r \quad=$ distance de la cible au point de calcul (m)

$x=$ épaisseur des protections en $\mathrm{g} / \mathrm{cm}^{2}$,

$\lambda=$ parcours moyen en $\mathrm{g} / \mathrm{cm}^{2}$.

Les calculs ont tous été effectués en des points situés juste derrière les protections.

Les murs sont, pour la plupart, constitués de béton standard, mais, par souci d'économie, nous avons par endroit remplacé le béton par de la terre, ailleurs, au contraire, pour gagner de la place, nous avons utilisé un matériau plus lourd tel le béton baryté. 
TABLEAU I

Valeurs de $H_{0}$ et de $\lambda$ en fonction de l'angle d'incidence du faisceau par rapport au point de calcul de la protection $H_{0}$ and $\lambda$ values as a function of the angle between the beam axis and the point of calculation in the shield

\begin{tabular}{|c|c|c|c|}
\hline Angle & $0^{\circ} \mathrm{a} 30^{\circ}$ & $30^{\circ} \cong 60^{\circ}$ & $\begin{array}{l}90^{\circ} \\
\text { tectionlate }\end{array}$ \\
\hline$H_{0}$ & $1,52 \quad 10^{-15}$ & $0,3810^{-15}$ & $1,410^{-17}$ \\
\hline$\lambda$ béton & 56 & 48 & 40 \\
\hline$\lambda$ terre & 56 & 48 & 40 \\
\hline$\lambda$ béton baryté & 65 & 56 & 44 \\
\hline$\lambda \mathrm{fer}$ & 85 & 73 & 61 \\
\hline
\end{tabular}

\subsection{Calcul des labyrinthes}

Ces labyrinthes permettent l'accès aux salles de traitement en évitant les lourdes portes en béton ou en fer difficiles d'utilisation en fonctionnement médical. Il s'agit d'une succession de couloirs faisant un angle entre eux et dont le but est d'augmenter la diffusion des particules secondaires et les épaisseurs d'atténuation.

Pour calculer la dose équivalente en sortie du labyrinthe, on utilise la formule de Tesch [11]:

$$
H\left(r_{1}, r_{2}, r_{3}\right)=\frac{H_{a}}{r_{1}^{2}} r \times 2 a^{2} \times f_{2}\left(A_{2}, r_{2}\right) \times f_{3}\left(A_{3}, r_{3}\right)
$$

- $f_{2}$ et $f_{3}$ sont respectivement des fonctions des sections $A_{2}$ et $A_{3}$ des différentes parties du labyrinthe et de leurs longueurs $r_{2}$ et $r_{3}$.

- $H_{a}$ est l'équivalent de dose à l'entrée du labyrinthe (Sv/proton)

- $a$ et $r$ sont respectivement les distances en mètre de la source à l'entrée du labyrinthe et les longueurs des différentes sections (Figs. 1 et 2).

\subsection{Modalités de fonctionnement du cyclotron}

Le champ des particules secondaires émises est, entre autres, fonction de l'intensité du faisceau de protons susceptibles de heurter les différentes structures du cyclotron. Pour les calculs de radioprotection, nous avons pris comme hypothèse de base que des intensités de $50 \mathrm{nA}$ et de $20 \mu \mathrm{A}$ seraient respective- 
ment utilisées pour les traitements avec les proton et les neutrons. Nous avons choisi l'option la plus pessimiste qui est de perdre $5 \%$ de l'intensité du faisceau de protons à $65 \mathrm{MeV}$ dans le cyclotron et $10 \%$ dans la voie de faisceau.

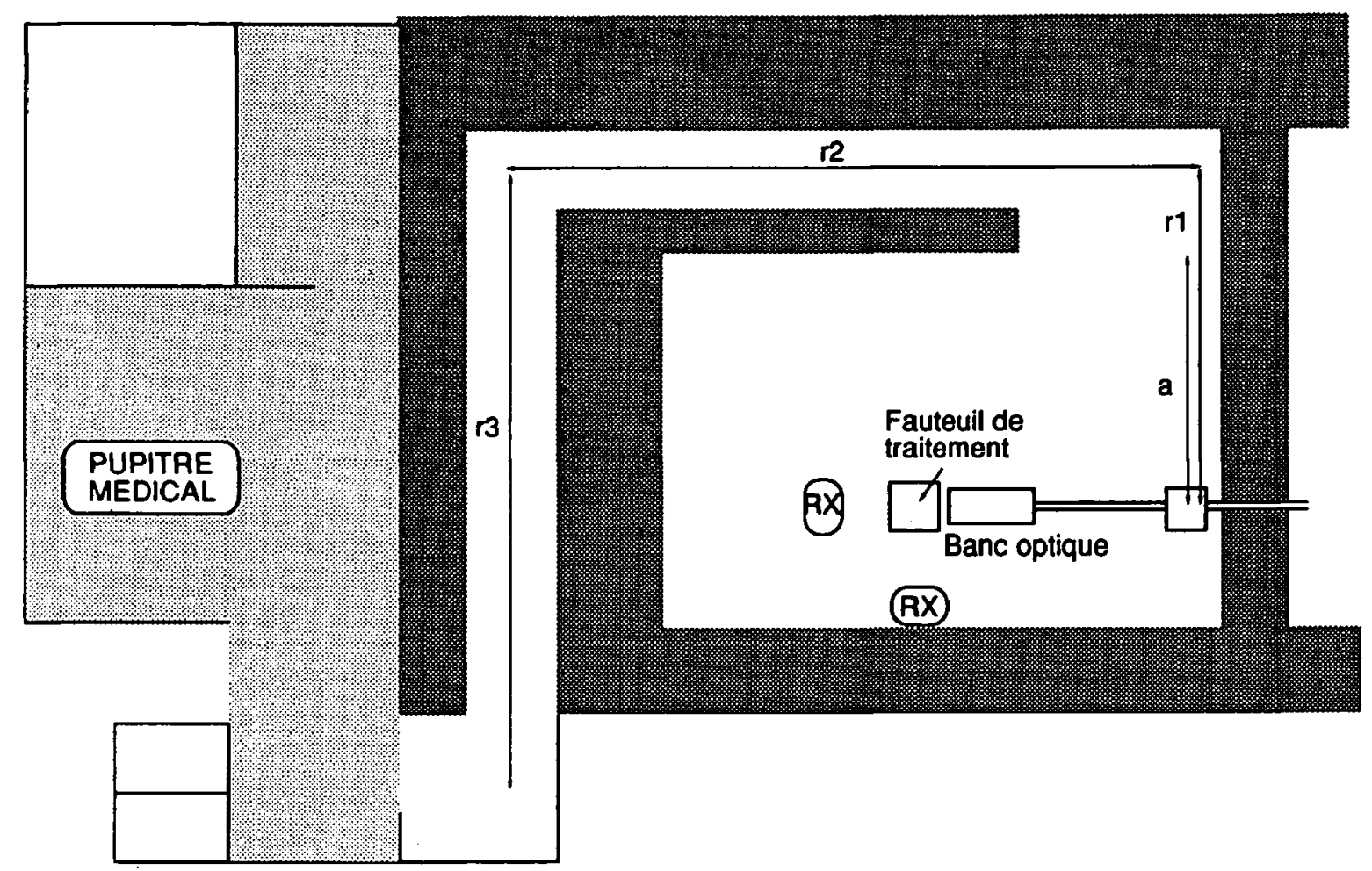

Fig. 1 - Salle de protonthérapie.

Protontherapy treatment room.

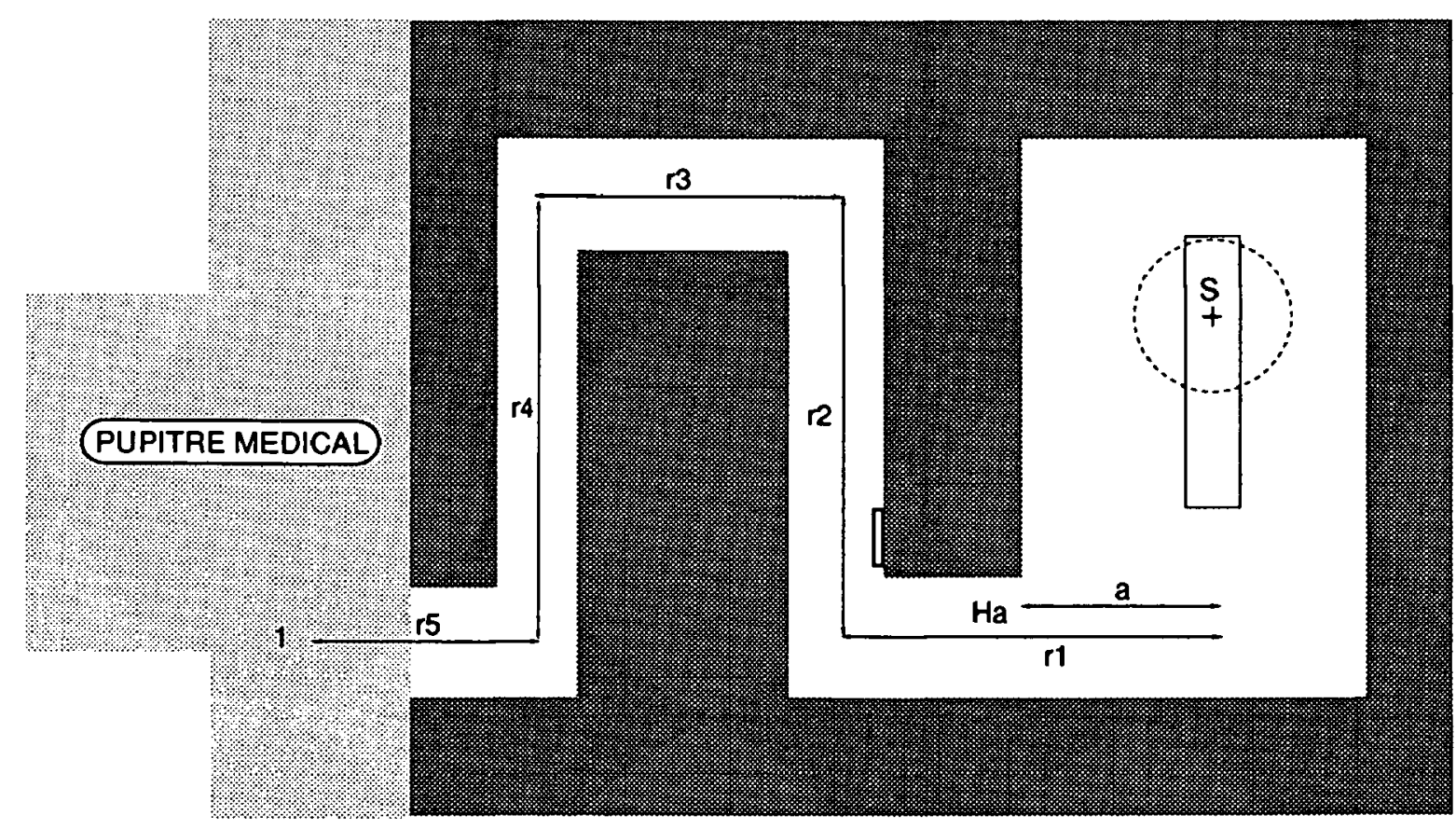

Fig. 2 - Salle de neutronthérapie

Neutrontherapy treatment room. 


\section{Epaisseur des murs et débits de dose prévus}

\subsection{Autour du cyclotron}

Une épaisseur de $600 \mathrm{~g} / \mathrm{cm}^{2}$ soit $250 \mathrm{~cm}$ de béton assure dans les zones 1,2 et 3 (Fig. 3) des débits de dose inférieurs à 2,5 $\mu \mathrm{Sv} / \mathrm{h}$. La même épaisseur donne à la verticale du cyclotron, à $8 \mathrm{~m}$ du centre, un débit de $3 \mathrm{nSv} / \mathrm{h}$. Dans la salle de contrôle le débit sera encore inférieur. La porte d'accès au cyclotron de 2,5 m d'épaisseur est celle qui est habituellement utilisée dans les accélérateurs de haute énergie.

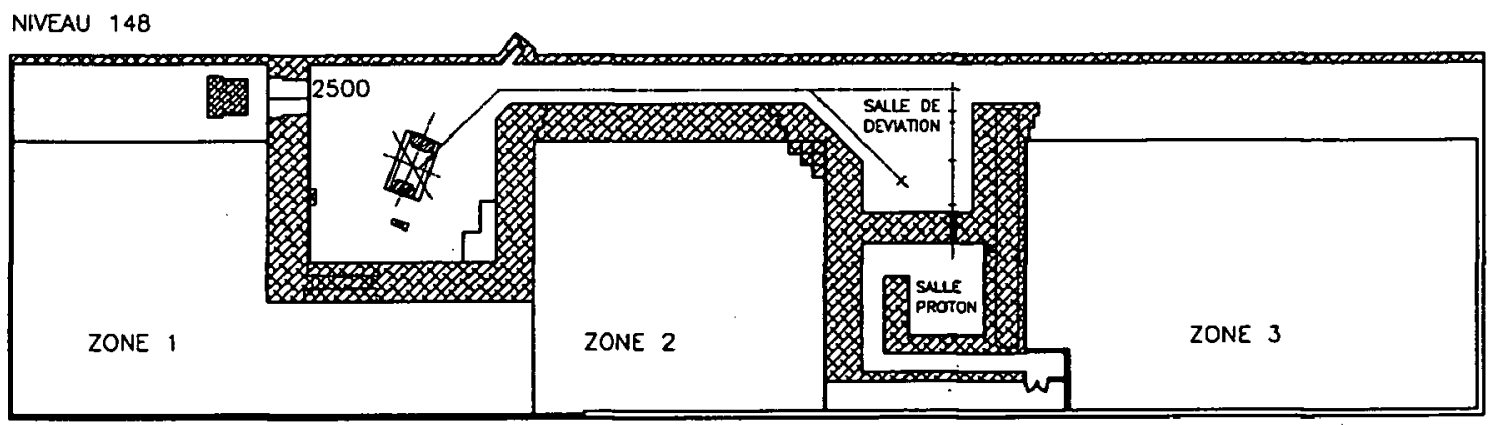

NIVEAU 144

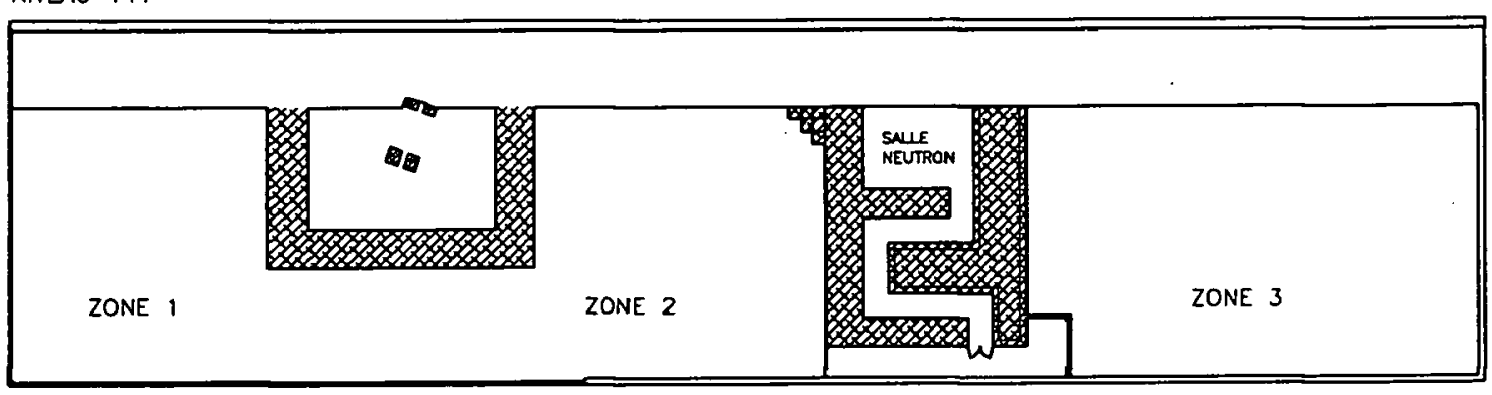

Fig. 3 - Implantation du cyclotron biomédical MEDICYC.

Representation of the MEDICYC biomedical cyclotron building.

\subsection{Dans la voie de faisceau}

$240 \mathrm{~cm}$ de béton donne, à $4 \mathrm{~m}$ derrière les murs, de l'ordre de $50 \mathrm{nSv} / \mathrm{h}$ avec des pics maximaux de $0,3 \mu \mathrm{Sv} / \mathrm{h}$ dans le court instant où $20 \mu \mathrm{A}$ de protons sont arrêtés sur une cage de Faraday. A l'extrémité de la voie de faisceau, les $4 \mathrm{~m}$ de béton réduisent le débit à $1,5 \mu \mathrm{Sv} / \mathrm{h}$ dans le cas le plus improbable où $20 \mu \mathrm{A}$ de protons y seraient arrêtés accidentellement. Au-dessus de la salle de déviation, 3,2 m de terre assurent une protection équivalente à 2,4 $\mathrm{m}$ de béton.

Il faut noter que les protections sont constituées de blocs mobiles dont l'agencement en quinconce a été calculé de telle sorte que les joints entre blocs soient discontinus. L'avantage de ces blocs est de permettre, éventuellement, une organisation différente en vue, par exemple, de la création d'autres salles 
de traitement. Partout où les flux de neutrons sont prévus ponctuellement plus importants que les hypothèses de calcul (cible de béryllium pour la neutronthérapie, cage de Faraday...), des protections rapprochées en fer seront réalisées.

\subsection{Dans la salle de neutronthérapie}

Le flux de neutrons obtenu dans ce faisceau vertical fixe est parfaitement collimaté puisqu'il sert au traitement des patients. Des murs de $240 \mathrm{~cm}$ de béton sont suffisants pour assurer un débit de dose, à l'extérieur de la salle, inférieur à $0,3 \mu \mathrm{Sv} / \mathrm{h}$. Le labyrinthe assure au pupitre médical un débit de dose inférieur à $0,2 \mu \mathrm{Sv} / \mathrm{h}$. Les neutrons émis sont piégés dans un puits de $3 \mathrm{~m}$ de profondeur, ce qui évite le rayonnement rétro-diffusé par le sol qui est un risque d'irradiation inutile pour le malade.

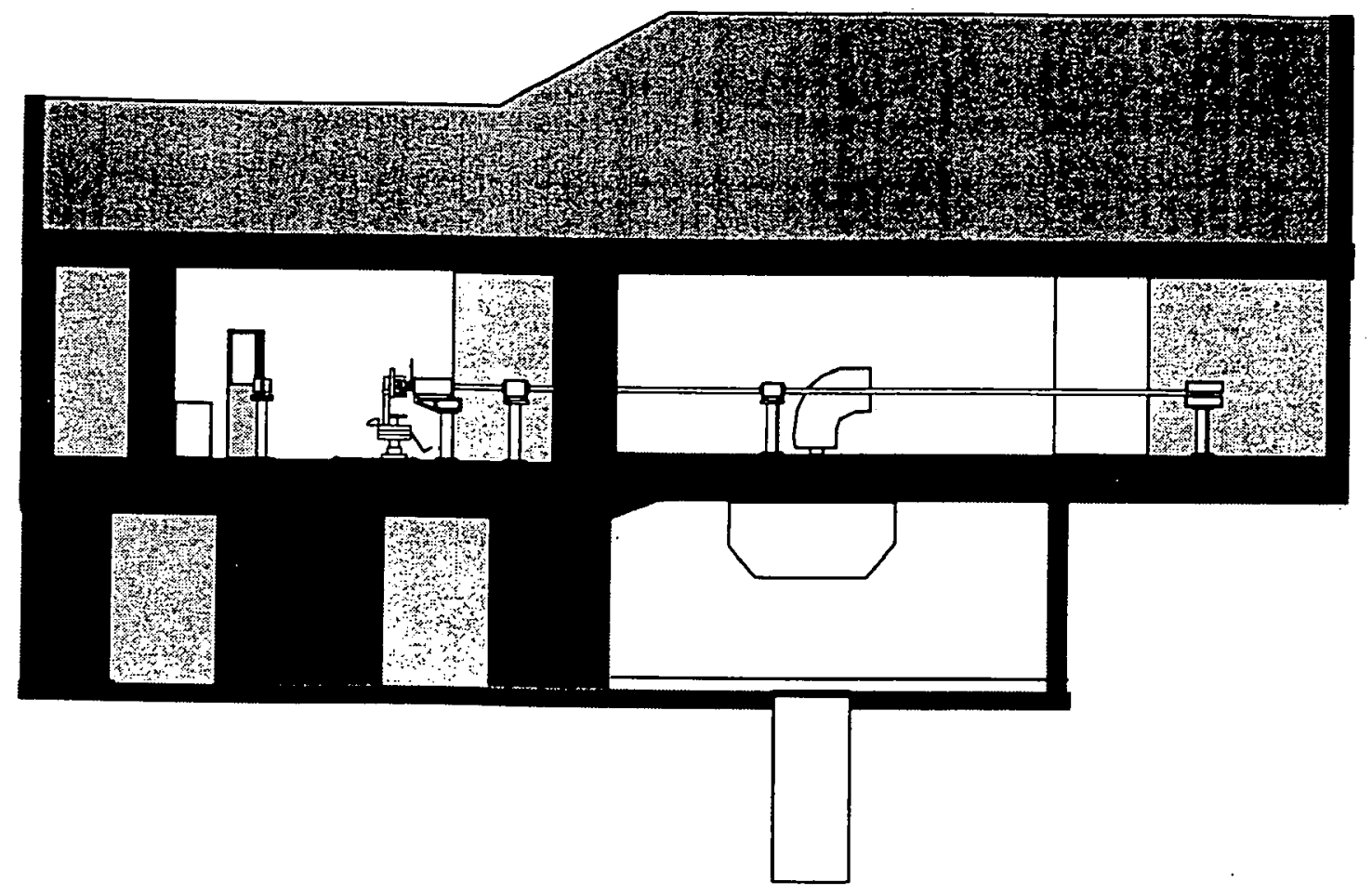

Fig. 4 - Implantation du cyclotron biomédical : coupe transverse.

Representation of the biomedical cyclotron : cross view.

\subsection{Dans la salle de protonthérapie}

Pour des raisons pratiques de construction et compte tenu de l'incertitude qui règne encore sur l'attribution qui sera faite pour les zones environnantes, un maximum de précautions a été pris et l'épaisseur des murs est ici aussi l'équivalent de $240 \mathrm{~cm}$ de béton.

D'autre part, la disposition des deux salles de protonthérapie et de neutronthérapie est telle (Fig. 4) que $80 \mathrm{~cm}$ d'épaisseur de plafond pour la salle neutron et $120 \mathrm{~cm}$ d'épaisseur de sol pour la salle protons sont suffisants pour permettre l'accès dans une salle quand des traitements se déroulent dans l'autre. 


\section{Exposition en limite du site}

Une mesure de la radioactivité ambiante a été réalisée sur le site de la construction du bâtiment avant le début des travaux et des prélèvements de terre et d'eau ont été analysés au CERN. Le but de ces mesures était de posséder un document de référence concernant l'activité naturelle du site avant la mise en route du cyclotron. Le débit de dose naturel varie entre 74 et $136 \mathrm{nSv} / \mathrm{h}$ pour les photons et muons, il est de $7 \mathrm{nSv} / \mathrm{h}$ pour les neutrons, tandis que l'activité bêta totale pour les aérosols est de $0,97 \mathrm{mBq} / \mathrm{m}^{3}$.

La limite du site la plus proche du cyclotron est à $45 \mathrm{~m}$ du centre de ce dernier ; en se basant sur le fonctionnement normal du cyclotron tel que décrit plus haut, en tenant compte du temps d'utilisation et en se référant au débit de dose trouvé à $10 \mathrm{~m}$ derrière les murs du cyclotron, le débit de dose maximal serait de $56 \mathrm{nSv} / \mathrm{h}$, soit $40 \mu \mathrm{Sv} / \mathrm{an}$; cette valeur est surestimée car elle ne tient compte ni de l'atténuation des neutrons sur les murs du bâtiment, ni de la structure du terrain. Cette valeur reste faible par rapport à l'exposition naturelle locale.

\section{Activation de l'air}

\subsection{Hypothèses de calcul}

L'activation de l'air est surtout due aux réactions des neutrons avec les différents constituants de l'air. Les débits de dose relatifs à cette activation ont été évalués en utilisant les méthodes de calcul et les hypothèses développées par Sullivan [10] et Birattari [2]. Les principales réactions d'activation de l'air pour l'énergie de notre cyclotron sont résumées dans le tableau III. Nous avons supposé qu'aucun de ces radionucléides n'est métabolisé et que la dose à laquelle ils contribuent est uniquement due à une irradiation externe.

Le temps pendant lequel le faisceau est présent dans la salle du cyclotron, donc susceptible d'activer l'air, est directement lié au temps de traitement des malades. Des expériences de physique et de radiobiologie auront lieu dans le temps qui n'est pas imparti à la médecine, mais il est toutefois réaliste d'estimer pour les calculs que le temps global réel de fonctionnement journalier du cyclotron sera d'environ 3 heures. Bien entendu, l'air des salles de traitement est aussi activé et, compte tenu de leurs spécificités, le problème de l'activation de l'air sera prépondérant dans la salle de neutronthérapie.

\subsection{Ventilation de l'installation}

Compte tenu de leur volume, le débit de renouvellement de l'air sera de 6 volumes d'air par heure pour les salles de traitement et la voie de faisceau et de 4 volumes d'air par heure pour le cyclotron. Cet air aspiré des salles de traitement vers la voie de faisceau séjourne un quart d'heure dans la salle du cyclotron, afin de permettre la décroissance de certains isotopes $\left({ }^{16} \mathrm{~N}\right)$, avant d'être évacué dans l'atmosphère par une cheminée où l'activité pourra être contrôlée. 
TABLEAU III

Principales réactions d'activation de l'air autour du cyclotron et dans les salles de traitement

Main reactions of air activation around the cyclotron and the treatment rooms

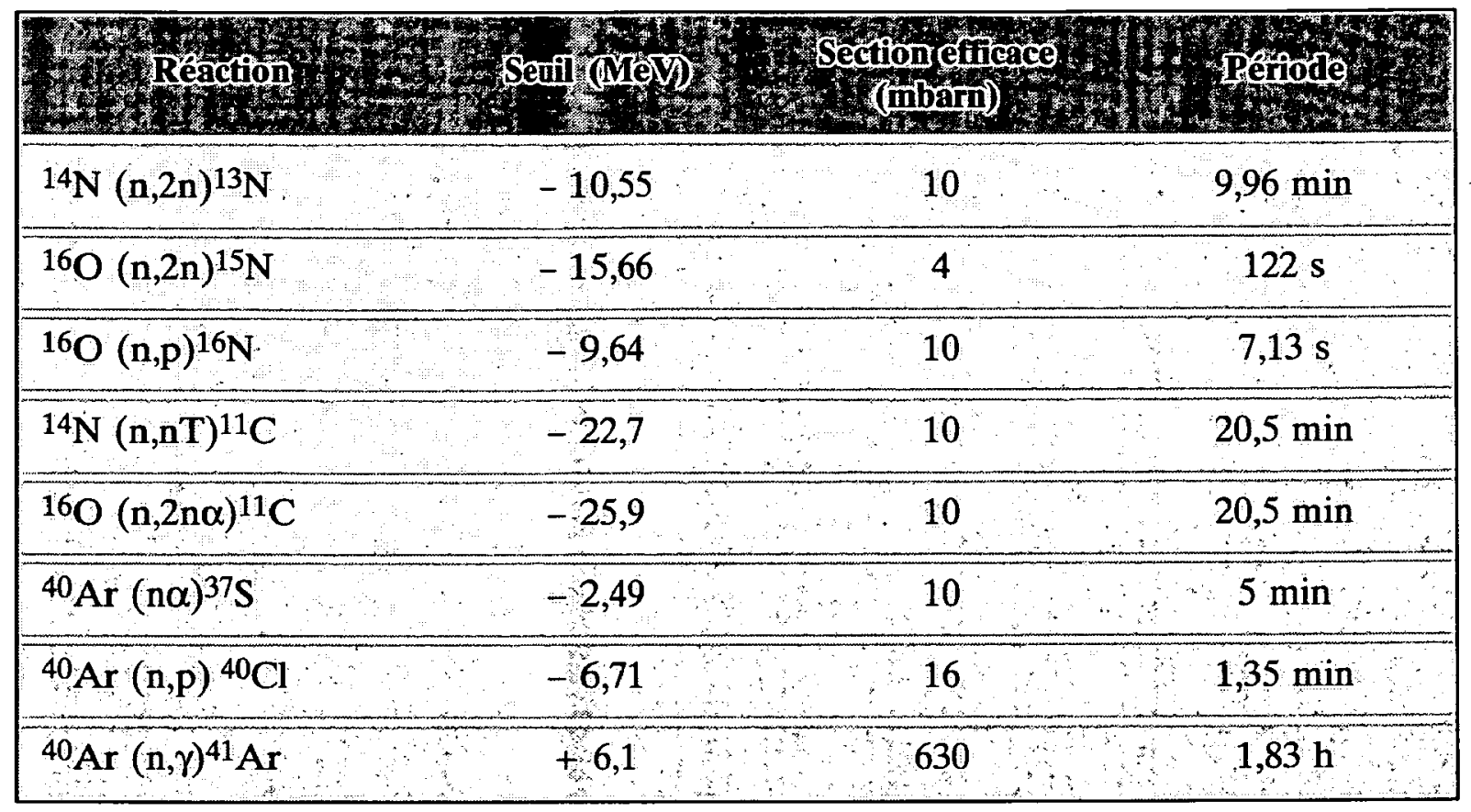

\subsection{Résultats}

Les calculs effectués dans ces conditions donnent, pour tous les radionucléides obtenus, des activités dans l'air inférieures à $410^{4} \mathrm{MBq} / \mathrm{h}$, ce qui, d'après Sullivan [10], entraîne une irradiation très faible. L'isotope dont l'activité moyenne est la plus élevée est $16 \mathrm{~N}$ dont la période de $7,13 \mathrm{~s}$ fait qu'il est éliminé très rapidement compte tenu de la ventilation des salles. Les principaux radionucléides présents en limite du site sont résumés dans le tableau IV.

\section{Exposition du personnel}

La dose que le personnel est susceptible de recevoir est difficile à prévoir. Pour le personnel technique, elle dépendra du nombre des interventions à effectuer sur la machine, de leur durée, du lieu de l'intervention (zone très active ou pas) et du temps de désactivation que l'on peut ou non respecter avant l'intervention. Pour le personnel médical, cette exposition sera négligeable dans la salle de protonthérapie mais pas dans la salle de neutronthérapie ; elle dépendra non seulement de l'énergie des neutrons émis mais aussi du nombre de patients traités, du nombre de faisceaux par patient, du temps d'attente entre la fin du traitement et l'entrée dans la salle pour dégager le patient, mais aussi du nombre de manipulateurs présents par salle et se partageant donc le travail ; l'exposition est aussi fonction de la qualité de l'installation: l'irradiation du personnel étant plus importante avec l'utilisation des 
TABLEAU IV

Principaux radionucléides présents en limite du site Main radionuclides present at the cyclotron limits

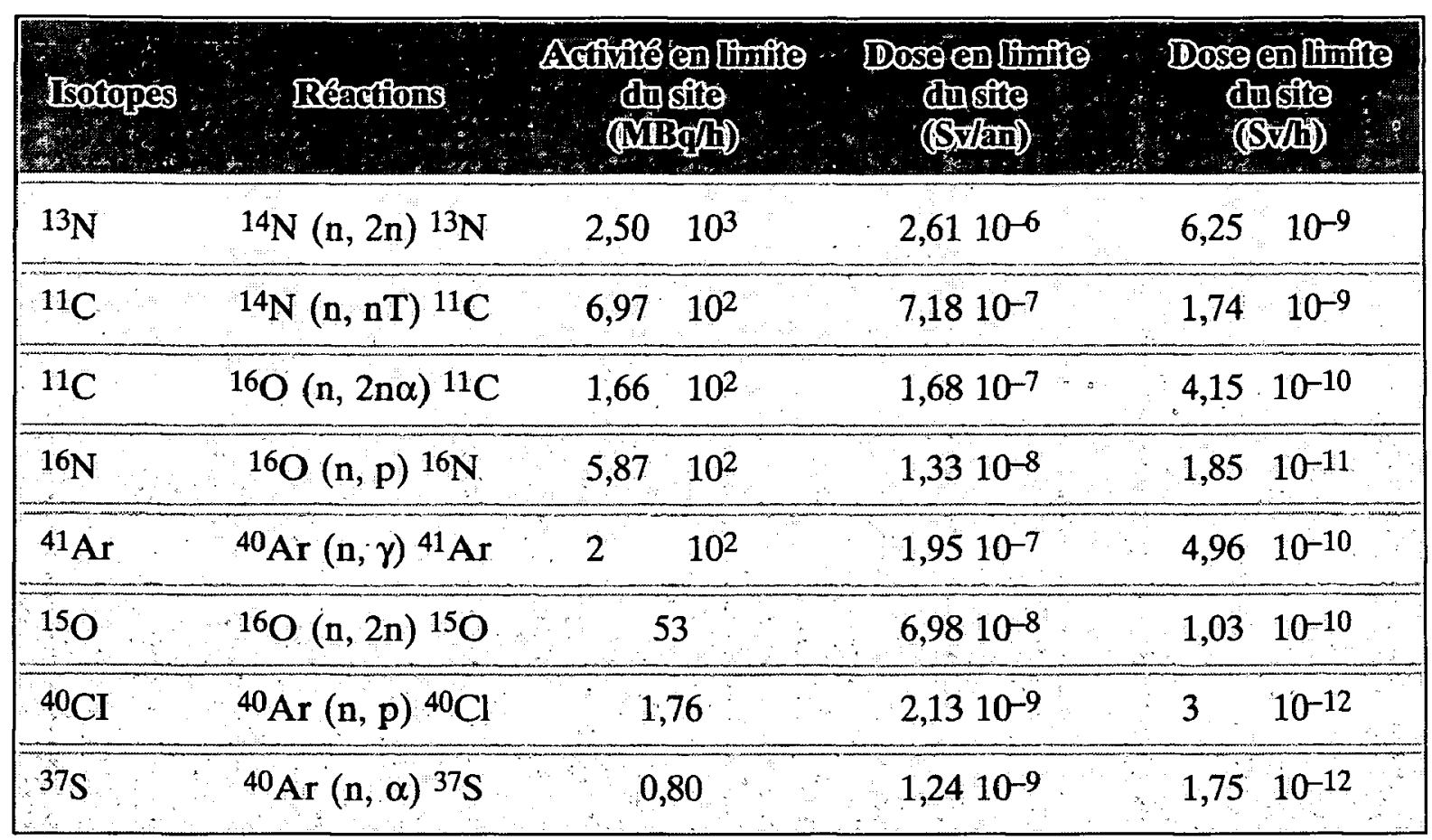

anciens collimateurs à ouverture fixe et devant donc être manipulés souvent qu'avec des collimateurs intégrés à la tête de traitement et dont l'ouverture est commandée à distance. Compte tenu de tous ces facteurs, les données rapportées par les différents auteurs ne sont pas très homogènes. Les équipes d'Orléans en France, de Louvain La Neuve en Belgique, de Clatterbridge en Angleterre, de Seattle et de Fermilab aux USA ont une grande expérience de traitement par neutronthérapie avec des machines d'énergie à peu près identiques mais des installations très différentes : Fermilab $\{p(66)+\mathrm{Be}\}$ [8], indique une dose de $5 \mu \mathrm{Sv}$ et Seattle $\{\mathrm{p}(48)+\mathrm{Be}\}$ [4] $4 \mu \mathrm{Sv}$ par champ traité ; ces résultats sont comparables aux $5,1 \mu \mathrm{Sv}$ par champ de Clatterbrige [5] ; à Louvain [13] la dose par patient est de $150 \mu \mathrm{Sv}$ mais il y a plusieurs champs par patient et, ici, il n'y a qu'une seule manipulatrice. A Nice, nous n'avons pas suffisamment de recul en traitement par neutrons pour pouvoir donner des conclusions concernant l'irradiation de l'équipe médicale, mais une étude systématique de l'activité réelle dans la salle de neutronthérapie concernant à la fois l'air, le collimateur, la table de traitement et le patient doit être entreprise et fera l'objet d'une autre communication.

\section{Conclusion}

Le but de cette note était de décrire la méthode utilisée pour le calcul des épaisseurs des protections et l'évaluation de l'activation de l'air et des struc- 
tures autour d'un cyclotron. Nous n'avons pas détaillé toutes les consignes de sécurité mais la radioprotection du personnel exige des règles très strictes de fonctionnement tant pour l'équipe technique que médicale : accès au hall du cyclotron ou aux salles de traitement, mise en route du cyclotron, demande du faisceau dans les salles de traitement et interventions sur l'ensemble de l'installation. Des balises de radioprotection reliées à une signalisation lumineuse autorisent ou non l'accès au hall du cyclotron, à la voie de faisceau et aux salles de traitement et donnent un état de l'activité de ces différentes zones; de même un système de clés ne permet le fonctionnement que sous certaines conditions. Après plus de trois ans de fonctionnement en protonthérapie et un an en neutronthérapie, les dosifilms $\beta, \gamma$ et neutron tant du personnel médical que technique lus par l'Office de protection contre les rayonnements ionisants (OPRI) n'ont pas mis en évidence de dépassement de dose par rapport aux limites recommandées.

\section{RÉFÉRENCES}

[1] AGENCE INTERNATIONALE DE L'ENERGIE ATOMIQUE (AIEA) - Radiological safety aspects of the operation of proton accelerator. (Technical report series $n^{\circ} 283$ ). Vienne : AIEA, 1988.

[2] BIRATTARI C., BONARDI M., FERRARI A., SILARI M. - Neutron activation of air by a biomedical cyclotron and an assessment of dose to neighbourhood population. Radiat. Prot. Dosim., 1986, 14, 311-319.

[3] BRAID T.H., RAPIDS R.F., SIEMSSEN R.H., TIPPIE J.W., O'BRIEN K. - Calculation of shielding for large cyclotrons. IEEE Trans. Nucl. Sci., 1971, NS-18, p. 821.

[4] EENMAA J., WOOTTON P., RISLER R. - Technologists exposures and radiation protection aspects of the clinical neutron therapy facility in Seattle. Br. J. Radiol., 1987, 60, p. 310.

[5] FINCH J., BONNET D.E. - An investigation of the dose equivalent to radiographers from a high-energy neutron therapy facility. Br. J. Radiol., 1992, 65, 327-333.

[6] FRANCE. Protection contre les rayonnements ionisants. Décret $n^{\circ} 86-1103$ du 2 octobre 1986. J.O.R.F., 12 octobre 1986. In : Protection contre les rayonnements ionisants (Brochure $n^{\circ}$ 1420). Paris : Journal officiel, 1990, vol. 2, 427-453.

[7] PATTERSON H.W., THOMAS R.H. - Acceleration health physics. New York: Academic Press, 1973.

[8] ROSENBERG I., AWSCHALOM M., TEN HAKEN R.K, BENNET B.R. - Analysis of personnel exposures in neutron therapy facilities. Health. Phys., 1984, 46(4), 407-412.

[9] STEVENSON G.R. - Shielding of low energy accelerators ( $<100 \mathrm{MeV})$ : Part I (Report HSRP/IR/80-51). Genève : CERN, 1980.

[10] SULLIVAN A.H. - Criteria for assessing the release of radioactive air from accelerators. (Report HS-RP/IR/82-26). Genève : CERN, 1982.

[11] TESCH K. - The attenuation of the neutron dose equivalent in a labyrinth through an accelerator shield. Part. Accel., 1982, 12, 169-175.

[12] TESCH K. - A simple estimation of the lateral shielding for proton accelerator in the energy range 50 to $1000 \mathrm{MeV}$. Rad. Prot. Dosim., 1985, 11(3), p. 165.

[13] VYNCKIER S.PIHET P., WAMBERSIE A. - Result of $10 \mathrm{y}$ of radiation protection dosimetry at the neutrontherapy facility in Louvain-La-Neuve. Health. Phys., 1989, 57(4), 529535 . 